\title{
Angle Trisection by Straightedge and Compass Only
}

\section{Nikolaos Vrettos Greece}

\begin{abstract}
For many centuries since ancient times the angle trisection has remained one of the three geometric problems that could not have a clear solution by straightedge and compass. Should such a solution had been, no one would have spent time on it. Moreover, no need would have been to prove that its solution is impossible due to the third degree equation. No need would have been to prove that it is impossible to construct a great number of regular polygons which now by this solution is absolutely possible. Furthermore, this solution by putting an end to all this, should also make us wonder whether Algebra can substitute Geometry especially when in an equation exist arithmetic factors behind which, a possibly different structure of it, is well hidden.
\end{abstract}

\section{I.The Solution}

Let $\mathrm{BOE}$ be an angle greater than or equal to 0 degrees and less than or equal to 180 degrees, with sides, the equal line segments OB and OE (Figures 1,2).

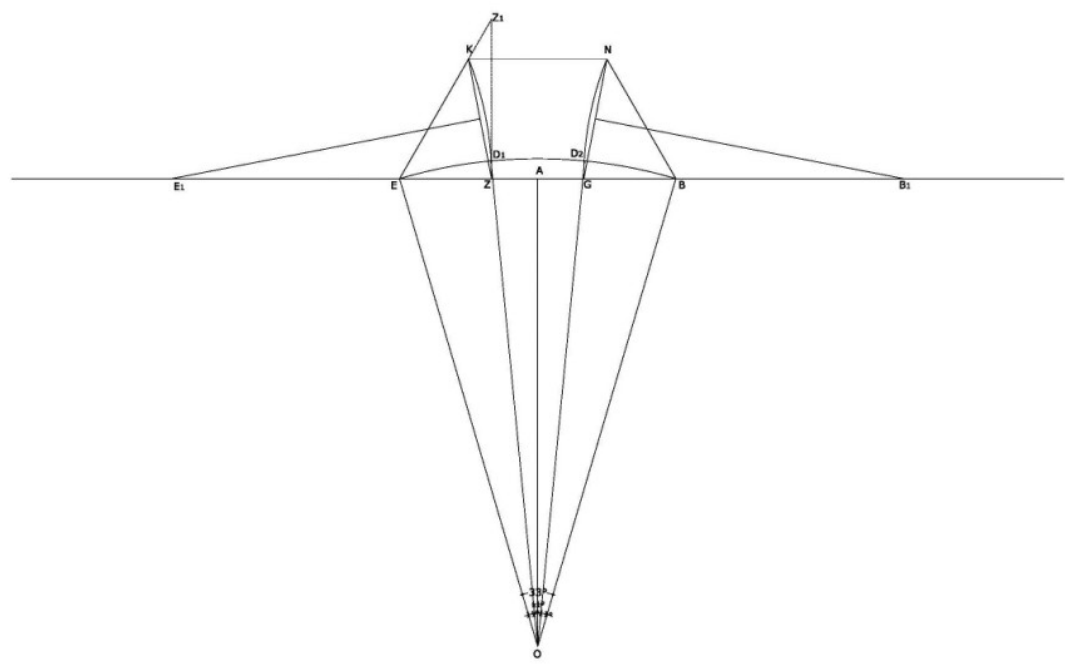

Figure 1

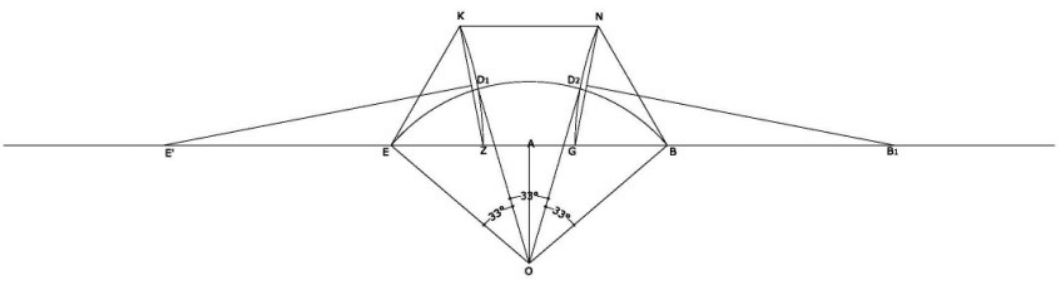

Figure 2

With center of the circle the point $\mathrm{O}$ and radius $\mathrm{OE}$, we write the arc EB and EB chord, the latter being

divided into two equal segments $\mathrm{EA}$ and $\mathrm{AB}$ and into three equal segments $\mathrm{EZ}, \mathrm{ZG}$ and $\mathrm{GB}$.

With center $\mathrm{A}$ and radius $\mathrm{AB}$ we write a circle and the equilateral triangles KEA and $\mathrm{NAB}$ and thus, half upper EKNB regular hexagon inscribed in circle (A,AB) (Figure 1).

From the middle of the line segments $\mathrm{KZ}$ and $\mathrm{NG}$ we raise perpendicular lines to either side extensions of the line segment EB which will intersect it at points E1 and B1.

With center E1 and radius E1Z we write circle and respectively with center B1 and 
radius $\mathrm{B} 1 \mathrm{G}$ we write again a circle; the circumferences of these circles will intersect the circle circumference $(\mathrm{A}, \mathrm{AB})$ at points $\mathrm{K}$ and $\mathrm{N}$ which are the vertices of the regular hexagon, whose upper half part is shown in Figure 1. The points of intersection of these two circles (E1,E1Z) and $(\mathrm{B} 1, \mathrm{~B} 1 \mathrm{G})$ to the arc $\mathrm{EB}$ of the circle $(\mathrm{O}, \mathrm{OE})$ are the points $\mathrm{D} 1$ and $\mathrm{D} 2$.

We will prove that the chords ED1, D1D2 and D2B included in the arc EB of the circle $(\mathrm{O}$, $\mathrm{OE}$ ) are equal and therefore, the corresponding arcs ED1, D1D2 and D2B are equal. Consequently the angles D1OE, D2OD1 and BOD2 are equal, that is, random angle BOE has been trisected.

\section{II.Way of Proving}

We will prove that the equal sides $\mathrm{EK}, \mathrm{KN}$ and $\mathrm{NB}$ of the regular hexagon, compared one by one and in order with the segments ED1, D1D2 and D2B (that is EK with ED1, KN with $\mathrm{D} 1 \mathrm{D} 2$ and NB with $\mathrm{D} 2 \mathrm{~B})$ are reduced by the same segment length ie that $\mathrm{ED} 1=\mathrm{D} 1 \mathrm{D} 2=\mathrm{D} 2 \mathrm{~B}$.

But since D1, D2 and B are points of the same arc EB of the circle $(\mathrm{O}, \mathrm{OE})$ and ED1, D1D2 and D2B are chords of arcs of the same circle, then respectively the angles BOD2, D2OD1 and D1OE will also be equal, as going through equal arcs.

\subsection{Steps of Proof}

1. We prove that $\mathrm{K}$ is the midpoint of the segment $\mathrm{S} 1 \mathrm{Z} 1$.

2. We prove that the triangles $Z 1 F X 1, S S 1 \mathrm{~V}$ are equal so $Z 1 F=S S 1$ and therefore because the first step proved that $\mathrm{K}$ is the midpoint of $\mathrm{S} 1 \mathrm{Z1}$, it becomes clear that $\mathrm{SK}=\mathrm{KF}$ and $\mathrm{SK}=$ $\mathrm{SY} 1=\mathrm{DD} 1$.

3. Because $\mathrm{ES}=\mathrm{ED} 1$ and because $\mathrm{ES}=(\mathrm{EK})-(\mathrm{SK})$ where SK represents the reduction of the length of the side of the original regular hexagon in order to be ED1 = ES, we prove that $\mathrm{ED} 1=\mathrm{D} 1 \mathrm{D} 2$ and that $\mathrm{D} 1 \mathrm{D} 2$ is equal to $\mathrm{KN}$ (which is equal to EK) reduced by the length $(\mathrm{MD} 1)+(\mathrm{D} 2 \mathrm{M} 1)=\mathrm{DD} 1$, i.e. $\mathrm{ED} 1=\mathrm{D} 1 \mathrm{D} 2$.

\section{III.Proof}

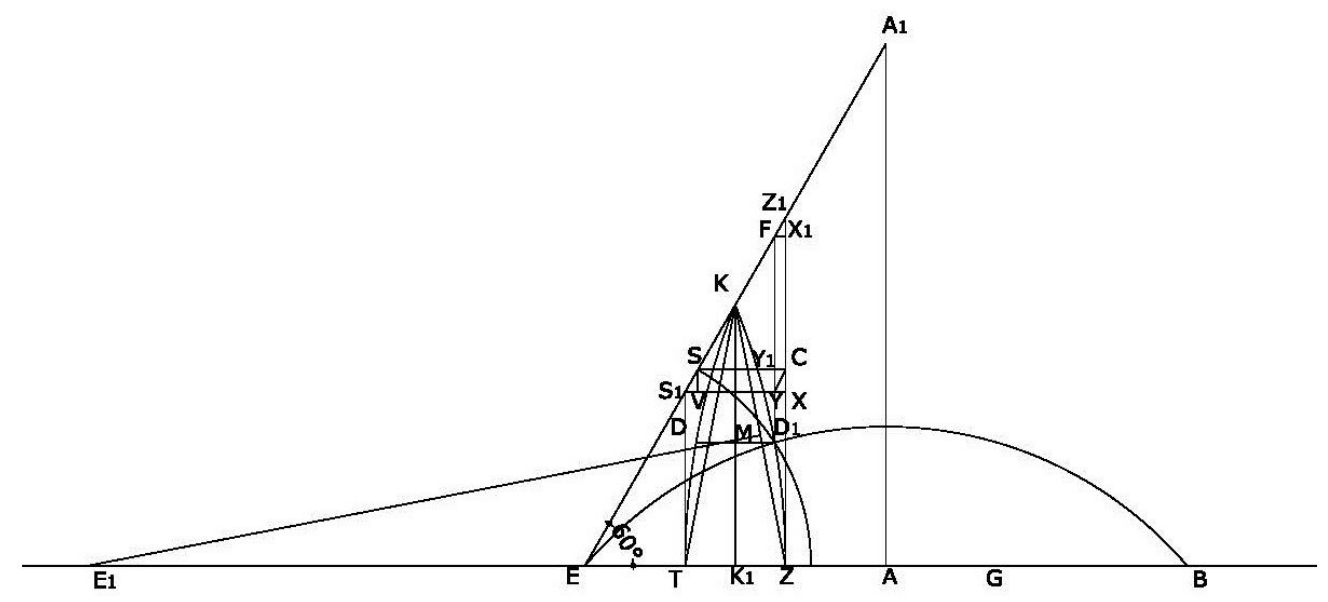

Figure 3

In Figure 3 we see the left part of the upper hexagon EKNB which we created.

Whatever we will prove about the random point D1 on the arc KZ, of the circumference of circle $(\mathrm{E} 1, \mathrm{E} 1 \mathrm{Z})$,

similarly applies for its symmetric arc NG to the right of line AA1.

From point $Z$ rises a perpendicular that will intersect the upward extension of side EK at point Z1.This

creates the right triangle $\mathrm{ZZ1E}$ with a $60^{\circ}$ degree angle at the vertex $\mathrm{E}$ and a $30^{\circ}$ degree angle at the vertex

$\mathrm{Z} 1$. So, side $\mathrm{EZ}=1 / 2(\mathrm{Z} 1 \mathrm{E})$ and $\mathrm{S} 1$ the midpoint of $\mathrm{Z} 1 \mathrm{E}$. With center the point $\mathrm{E}$ and radius 
EZ we write the arc ZS1 and from point S1 we draw a parallel line to EZ which will intersect $\mathrm{ZZ1}$ at point $\mathrm{X}$. In the right

triangle $\mathrm{XZ1S} 1$, the side $\mathrm{S} 1 \mathrm{X}=1 / 2(\mathrm{Z} 1 \mathrm{~S} 1)$. (Angle of $60^{\circ}$ degrees at vertex $\left.\mathrm{S} 1\right)$

By definition of the line segments EA and EZ, their difference is equal to $1 / 6$ of EB. But since $\mathrm{EK}=\mathrm{EA}$

and $\mathrm{EZ}=\mathrm{ES} 1$, the segment $\mathrm{S} 1 \mathrm{~K}=1 / 6(\mathrm{~EB}) . \quad$ In addition $\mathrm{EZ}=1 / 2(\mathrm{EZ} 1)=\mathrm{ES} 1=\mathrm{S} 1 \mathrm{Z} 1$ and by definition

$\overline{\mathrm{EZ}}=1 / 3(\mathrm{~EB})$ so $\mathrm{S} 1 \mathrm{Z} 1=2 / 6(\mathrm{~EB})$. However because $\mathrm{S} 1 \mathrm{~K}=1 / 6(\mathrm{~EB})$, the point $\mathrm{K}$ is the midpoint of $\mathrm{S} 1 \mathrm{Z} 1$.

From the point $\mathrm{K}$ we bring a perpendicular to $\mathrm{EZ}$ which will intersect it at point $\mathrm{K} 1$.

From the point $\mathrm{S} 1$ also a perpendicular to $\mathrm{EZ}$ which will intersect it at point $\mathrm{T}$.

The segments $\mathrm{TK} 1$ and $\mathrm{K} 1 \mathrm{Z}$ are equal because $\mathrm{S} 1 \mathrm{~K}$ and $\mathrm{KZ1}$ are equal between the parallel lines $\mathrm{S} 1 \mathrm{~T}, \mathrm{KK} 1$ and Z1Z. From the point S1 we bring a perpendicular to ZZ1 which it will intersect at point $\mathrm{X}$.

We create the arc $\mathrm{KT}$ symmetrical to the arc $\mathrm{KZ}$ as to the axis KK1. Any any point D1 is equidistant with

its symmetric D, from the axis KK1 and the two symmetrical points are also equidistant from the perpendiculars to $\mathrm{EZ}$, i.e. Z1Z and $\mathrm{S} 1 \mathrm{~T}$. That is, points $\mathrm{D}$ and $\mathrm{D} 1$ equidistant from $\mathrm{S} 1 \mathrm{~T}$ and $\mathrm{ZZ} 1$ are on a straight line, parallel to EA.

From point D1 we rise a perpendicular to intersect $\mathrm{S} 1 \mathrm{X}$ at point $\mathrm{Y}$ and extended upwardly it will intersect

EZ1 at point $\mathrm{F}$. Any point $\mathrm{D} 1$ of the $\operatorname{arc~} \mathrm{KZ}$ of the circle $(\mathrm{E} 1, \mathrm{E} 1 \mathrm{Z})$ is one and only one fixed length of distance

$\underline{\mathrm{D} 1 \mathrm{Y}}$ from $\mathrm{YX}$ and one and only one fixed length of distance D1L from ZZ1 and vice versa, i.e. the size of

D1L implies the size D1Y and vice versa. The same applies to the corresponding points symmetrical to the axis KK1.

We will prove that $\mathrm{SK}=\mathrm{SY} 1=\mathrm{DD} 1$.

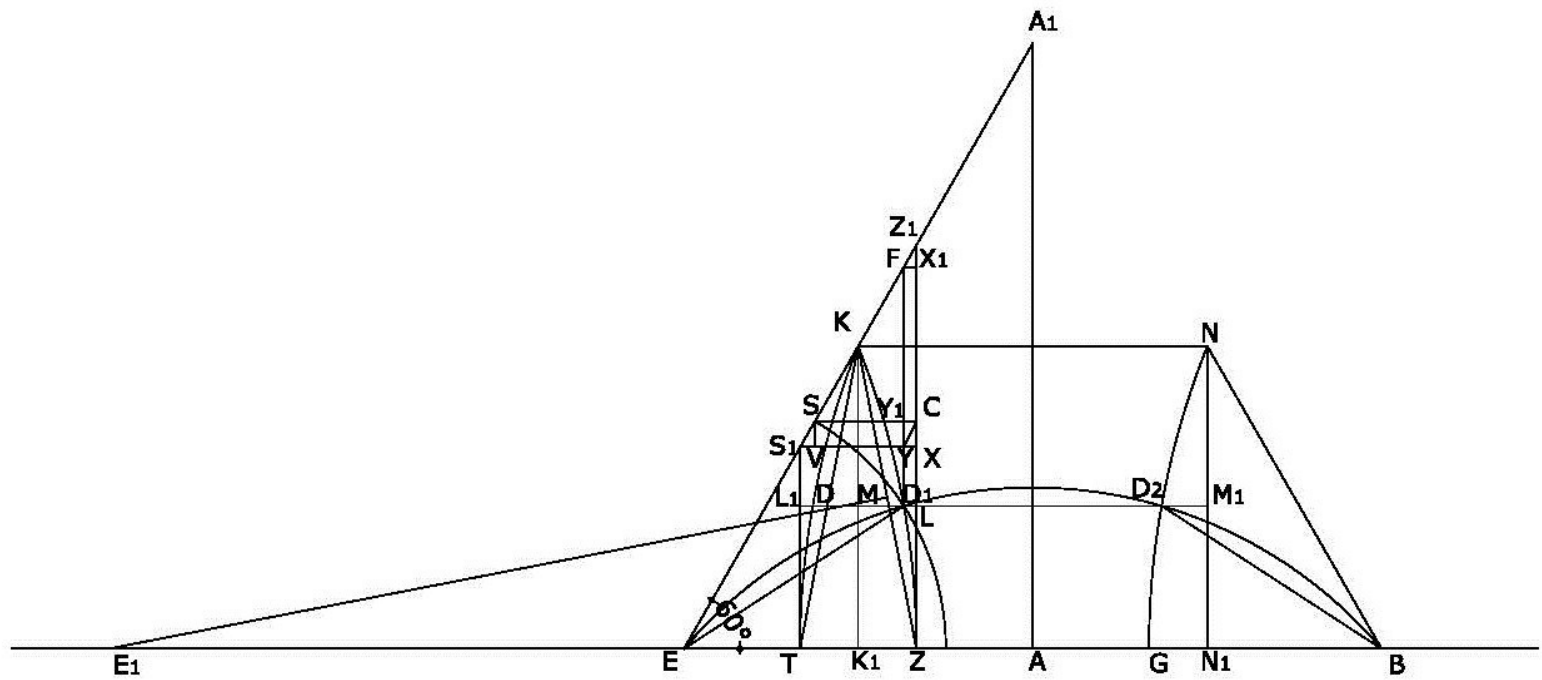

Figure 4

We join the vertex $\mathrm{K}$ of the regular hexagon with the point $\mathrm{A}$ to form the equilateral triangle KEA (Figure 4). The line KA will intersect D1F at the point Y1. From Y1 we draw a parallel to YX, 
which will intersect $\mathrm{KA}$ at point $\mathrm{C}$. This creates the rectangle YXCY1 with diagonal Y1X

From the point D1 we draw a parallel to EZ and perpendicular to ZZ1, which will intersect segments S1T,

$\mathrm{KK} 1$ and ZZ1 respectively at the points $\mathrm{L} 1, \mathrm{M}$ and $\mathrm{L}$. It will also intersect the arc $\mathrm{KT}$ at point D.

Because in the right triangle XZ1S1 the angles of vertices $\mathrm{Z} 1$ and $\mathrm{S} 1$ are $30^{\circ}$ degrees and $60^{\circ}$ degrees respectively and $\mathrm{K}$ is the midpoint of $\mathrm{S} 1 \mathrm{Z1}$, the triangle .KXZ1 is isosceles with base angles of $30^{\circ}$ degrees each. So, the right triangles $C Y 1 X$ and $\underline{X} 1 \mathrm{Z} \underline{\mathrm{F}}$ will be equal, as similar with equal vertical sides $\mathrm{F} \mathrm{X1}$ and $\mathrm{YIC}$. Respectively, $\mathrm{Y} 1 \mathrm{C}=\mathrm{Y} \mathrm{X}=\mathrm{D} 1 \mathrm{~L}=\mathrm{L} 1 \mathrm{D}=\mathrm{S} 1 \mathrm{~V}$ (point $\mathrm{D}$ symmetric to D1).And because the right triangles

CY1X and XCY are equal (Y1X and Y C diagonals of the rectangle Y XCY1) due to their similarity and

equality of their vertical sides $\mathrm{YX}$ and S1V and given that the lengths of the line segments D1L, D1Y, L1D

and DV are fixed and unique for point D1 and in relation to it, it follows that:

First, the points $\mathrm{S}, \mathrm{Y} 1$ and $\mathrm{C}$ will lie on a straight line because $\mathrm{SV}=\mathrm{Y} 1 \mathrm{Y}=\mathrm{CX}$ are the continuations of equal straight line segments DV, D1Y and LX.

Second, the triangles $\mathrm{X} 1 \mathrm{Z1F}$ and VSS1 are equal as equal to a third party (the triangle $\mathrm{XCY}$ ) so, $\mathrm{S} 1 \mathrm{~S}=\mathrm{FZ1}$ and therefore $\mathrm{SK}=\mathrm{KF}$.

Third, that the straight line segment SK as a side of the equilateral triangle KSY1 is equal to SY1 and the latter equal to DD1.

Essentially however, SK is the length representing the reduction of the sideEK of the regular hexagon up to point $S$, which lies on a circle with center point $E$ and radius $E D 1$, i.e. $E S=E D 1$, which means that from the equality $\mathrm{ES}=(\mathrm{EK})-(\mathrm{SK})$ and by the replacement of equal lengths we have $\mathrm{ED} 1=(\mathrm{EK})-(\mathrm{DD} 1)$. If from point $\mathrm{N}$ of the side $\mathrm{KN}$ of the regular hexagon we draw a perpendicular NN1 to EB and extend L1L to intersect NN1 at point M1, then, we make the segment $\mathrm{MM} 1=\mathrm{KN}$ where $\mathrm{M}$ is the midpoint of DD1.

Now, if the same procedure is repeated exactly on the symmetrical with axis AA1, right part of the regular hexagon on the equilateral triangle $\mathrm{NAB}$ at first we take the point $\mathrm{D} 2$ which is the respective intersection of two arcs as defined in the beginning and the segment D2M1 $=1 / 2$ $(\mathrm{DD} 1)=\mathrm{MD} 1$. But

since $\mathrm{MM} 1=(\mathrm{MD} 1)+(\mathrm{D} 1 \mathrm{D} 2)+(\mathrm{D} 2 \mathrm{M} 1)$, it follows that $\mathrm{D} 1 \mathrm{D} 2=(\mathrm{MM} 1)-(\mathrm{DD} 1)=(\mathrm{EK})-$ $(\mathrm{SK})=\mathrm{ED} 1$ which means that the random point $\mathrm{D} 1$, taken as defined in the beginning, creates the equal chords ED1 and D1D2 and the respective equal arcs and the respective equal angles $\mathrm{D} 1 \mathrm{OE}$ and D2OD1, i.e. the trisection of the random angle BOE has been accomplished.

\section{Acknowledgements}

Thanks to the architect Mrs Vaia Charizopoulos for drawing my figures in electronic form, trying really hard to approach the arc to arc intersections where needed. Finally, I must also thank Mr. Stefanos Mousafeiris for changing this paper in LaTeX form. 